\title{
Multidisciplinary Approach to Research of the Adherence to Treatment Phenomenon in Patients with Diabetes Mellitus
}

\author{
Anna Kogut ${ }^{1}$, M. Bobryk ${ }^{1}$, O. Potapov ${ }^{2}$, I. Frankova ${ }^{1}$, \\ J. Komisarenko ${ }^{1}$, O. Khaustova ${ }^{1}$, O. Chaban ${ }^{1}$ \\ ${ }^{1}$ Bogomolets National Medical University, Kyiv, Ukraine \\ ${ }^{2}$ Centre for Innovative Medical Technologies of the National Academy of Sciences of \\ Ukraine, Kyiv, Ukraine
}

Background: Diabetes Mellitus (DM) - is one of the most common chronic diseases, and its control is affected by the patients' psychological attributes (Esmaeilinasab et al., 2016). The number of patients with this disease is expected to increase in future. Given the increasing prevalence of DM, there is an urgent need for the treatment of DM and associated complications. Glycemic control largely depends on compliance with medication therapy. In fact, the most common problem in patients with DM - is a lack of medication compliance (Shamsi, Khodaifar, Arzaghi, Sarvghadi, Ghazi, 2014). For example, laboratory data of single components of the metabolic syndrome, according to International Diabetes Federation Consensus were dependent measures in multivariable regression models with self-reported alexithymia severity (TAS-20) and socio-demographic data. Alexithymia is a clinical trait, consisting of diminished introspective and interoceptive capacities that have been shown to implicate elevated autonomic outflow and to bias for hypertension (Lemche, Chaban, Lemche, 2014). One of the challenges in promoting adherence to medication regimes is having an accurate understanding of adherence rates and the factors that contribute to nonadherence (Lehmann et al., 2014). It's essential to consider the clinical assessment of psychological aspects in patients with DM, in order to prevent potentially adverse self-care management behaviours leading to diabetes-related complications, including declining levels of life quality (Conti et al., 2017). The patient`s quality of life is one of the principal components in clinical decision-making (Chaban, Khaustova, Bezsheiko, n.d.).

Purpose: Improving the effectiveness of DM patients' therapy and demonstrating the consequence of psychosocial factors on their adherence to treatment.

Method: This study was conducted during the period from July to August 2017 on the basis of the Kyiv Endocrinology Centre and the Department of Endocrinology in partnership with the Department of Medical Psychology, Psychosomatic Medicine and Psychotherapy of the Bogomolets National Medical University. Study population $(n=60)$ consisted of two comparative groups (CG) of patients with DM type 1 (DM1) $(n=16)$ and type 2 (DM2) $(n=33)$, while other patients had newly diagnosed DM of both DM1 and DM2. Study data consists of: hospital chart data (age, demographic data, body mass index, level of systolic and diastolic arterial pressure, blood glucose and glycated hemoglobin (HbA1c); psychometric validated scales (Medication Compliance Scale (MCS), Holmes and Rahe Stress Scale (HRSS), Dysfunctional attitudes Scale (DAS), Toronto Alexithymia Scale (TAS-20), the Depression, Anxiety and Stress Scale (DASS-21) and Chaban Quality of Life Scale (CQLS)). Glycemic control was assessed by HbA1c results. 
The statistical analysis was performed using descriptive statistics and Pearson's correlation with MS Excel and SPSS Statistics 22.0.

Results: A statistically significant difference was found between groups of patients with high (HC), middle (MC) and low level of compliance (LC) according to MCS. Patients with LC had statistically higher levels of HbA1c results according to MCS (LC (M=13), SD:1 vs. MC $(\mathrm{M}=9.83), \mathrm{SD}: 1.4$ vs. HC (M=9), SD:1.4). Average values by MCS results for CG - DM1 $(\mathrm{M}=19)$ and DM2 $(\mathrm{M}=18)$ present $\mathrm{MC}$; by HbA1c results - DM1 $(\mathrm{M}=10)$ and DM2 $(\mathrm{M}=10)$ at the same level. So, there was no significant statistical difference found between scores by the results in $\mathrm{CG}$.

A significant bilateral indirect correlation was found between the results by MCS (LC) with high level of the DAS, TAS-20, DASS-21, HRSS scores on level $p<0,001$ and direct by CQLS $(r=0,37)$ results on $p<0,01(\mathrm{HC})$. A relationship was also found between the scores by: 1) DAS (direct) - with the indicators of age, blood glucose level and depression, on level $\mathrm{p}<0,05$, alexithymia, stress and anxiety (DASS-21) on $\mathrm{p}<0,01 ; 2)$ CQLS (indirect) - with the DAS $(r=-0,28)$ and TAS-20 $(r=-0,29)$ results - on $\mathrm{p}<0,05$, stress $(r=-0,36)$ on $\mathrm{p}<0,01$ and depression (DASS-21) $(r=-0,48)$ on $p<0,001 ; 3)$ TAS-20 (direct) - with the DASS-21 subscales results on $\mathrm{p}<0,01$.

A strong relationship was found between LC in DM patients with high level scores of psychometric scales and laboratory indicators of HbA1c, as evidenced, that patients with LC most often had mental disorders and low rates of treatment effectiveness.

Conclusions: Adherence to treatment was not dependant on the type of diabetes and diagnosis. LC and quality of life added, in consequence, to non-adherence and, respectively, to the inefficiency of DM patients treatment. So, a multidisciplinary approach - involving psychologists for improving adherence to therapy together with endocrinology specialists, can potentially improve treatment effectiveness.

Originality: The comparison of DM patients indicators by psychosocial status, demographic data and level of compliance, depending on the type of diabetes and diagnosis.

Limitations of the study: The low sample of patients which is planned to be addressed in further studies.

Strengths of the study: The concept of "adherence to treatment" is much broader than the concept of "compliance", which is signified only by a strict adherence to the prescription of a doctor. Adherence to treatment - is a complex of the external and intrinsic factors of the patients' environment and their characteristics as personalities, in relation to medical treatment. There is an extensive scope for studying and researching both in both clinical and social science fields. Multidisciplinary research would be a recommended approach for future research, because different specialists in their distinctive spheres may be able to unite with the common purpose of studying the problem of comorbidity. 
Keywords: diabetes, health, health care, medical, medical research, medical science, medicine, mental health, mental illness, mental stress, psychology, quality of life, research, scientific research, social well-being.

\section{References.}

Chaban, O., Khaustova, O., Bezsheiko, V. (n.d.). Reliability and validity of Chaban Quality of Life Scale. Retrieved from https://www.ecnp.eu/presentationpdfs/70/P.2.h.301.pdf

Conti, C., Di Francesco, G., Fontanella, L., Carrozzino, D., Patierno, C., Vitacolonna, E., Fulcheri, M. (2017). Negative Affectivity Predicts Lower Quality of Life and Metabolic Control in Type 2 Diabetes Patients: A Structural Equation Modeling Approach. Front Psychol. 24;8:831. doi: 10.3389/fpsyg.2017.00831

Esmaeilinasab, M., Ebrahimi, M., Mokarrar, MH., Rahmati, L., Mahjouri, MY., Arzaghi, SM. (2016). Type II diabetes and personality; a study to explore other psychosomatic aspects of diabetes. J Diabetes Metab Disord. 3;15:54. doi: 10.1186/s40200-016-0281-3

Lehmann, A., Aslani, P., Ahmed, R., Celio J., Guchet A., Bedouch P., Bugnon O., Allenet B., Schneider MP. (2014). Assessing medication adherence: options to consider. Int J Clin Pharm. 36(1):55-69. doi: 10.1007/s11096-013-9865-x

Lemche, A., Chaban, O., Lemche, E. (2014). Alexithymia as a risk factor for type 2 diabetes mellitus in the metabolic syndrome: a cross-sectional study. Psychiatry Res. 215(2):438-43. doi: 10.1016/j.psychres.2013.12.004

Shamsi, F., Khodaifar, F., Arzaghi, SM., Sarvghadi, F., Ghazi, A. (2014). Is there any relationship between medication compliance and affective temperaments in patients with type 2 diabetes? (2014). J Diabetes Metab Disord. 27;13(1):96. doi: 10.1186/s40200-014-0096-z 